\title{
Primary Osteosarcoma of the Nasal Fossa: An Exceptional Presentation
}

\author{
Majdoul S*, Tawfiq N, Bouchbika Z, Benchakroun N,Jouhadi H, Saharaoui S and Benider A \\ Department of Oncology and Radiotherapy, Faculty of Medicine and Pharmacy, Hassan II University of Casablanca, \\ Morocco
}

*Corresponding author: Majdoul S, Department of Oncology and Radiotherapy, Faculty of Medicine and Pharmacy, Hassan II University of Casablanca, Morocco, Tel: 212-616068779, E-mail: salmajdoul@gmail.com

Citation: Majdoul S, Tawfiq N, Bouchbika Z, Benchakroun N, Jouhadi H, et al. (2017) Primary osteosarcoma of the nasal fossa: An exceptional Presentation. J Cancer Sci Clin Oncol 4(1): 104. doi: 10.15744/23946520.4.104

Received Date: March 08, 2017 Accepted Date: June 13, 2017 Published Date: June 22, 2017

\begin{abstract}
Sarcomas of the head and neck are very rare accounting for approximately $1 \%$ of all head and neck neoplasms. Primary osteosarcomas of the nasal cavity are less than $0.5 \%$ of the osteosarcomas occur in this location. Because of the rarity of this presentation, we report a case of a 54 year old male with primary osteosarcoma arising de novo from the nasal cavity, presented with left epistaxis and bilateral nasal obstruction.
\end{abstract}

We describe the clinical, pathological, treatment and outcome features of this rare tumor and provide a brief review of the literature.

Keywords: Osteosarcoma of fossa nasal; Rare case; Radiotherapy; Surgery; Chemotherapy

\section{Background}

Head and neck soft tissue sarcomas are a rare and heterogeneous group of mesenchymal neoplasms with more than 50 different histologic subtypes that account for only $2-15 \%$ of soft tissue sarcomas and $1 \%$ of head and neck tumors [1]. It occurs usually in bones of the jaw and has rarely been reported in the nasal cavity or paranasal sinuses [2]. Most soft tissue sarcomas display a similar natural history characterized by aggressive local growth with metastatic potential. However, the outcome of head and neck is generally worse compared to their non-head and neck counterparts, with lower rates of local control (74\% vs. $85 \%$, $\mathrm{p}<0.001)$ and disease-free survival $(64 \%$ vs. $76 \%, \mathrm{p}<0.001)[1]$.

The complex anatomy of the head and neck region makes these tumors challenging to manage, particularly the need for multimodality treatment and consideration for functional, esthetic, quality of life, and survival outcomes [2]. Surgery remains the mainstay treatment for head and neck sarcomas, and histological subtype becomes a less critical determinant for survival than the operability in terms of anatomic considerations, tumor size, and stage at diagnosis [1].

We report a rare case of an osteosarcoma in the nasal cavity of a 54 year-old male with clinical and histo-patholgical features.

\section{Case report}

We present the case of a 54-year-old male, without pathological history, who consulted for repeated left epistaxis, bilateral nasal obstruction and blurred vision in his left eye of over 8 months' duration, without exophthalmia. A nasal fibroscopy showed that both nostrils were completely occupied by a mass of polypoid appearance, friable and prone to haemorrhage infiltrating the nasopharynx. The ophthalmologic examination found no alterations of ocular motility or reflexes. Visual acuity was $6 / 10$ in the left eye and 10/10 in the right eye.

The cervico-facial CT with injection of gadolinium (Figure 1) revealed a mass occupying both nasal cavities, with destruction of the lamina papyracea of the left nostril and involvement of the left orbit, extending into maxillary sinus and prolapsed in the oropharynx.

An MRI was not performed because the patient carried a fixed metal prosthesis incompatible with the test. The PET analysis showed (Figure 2) a large mass occupying the nasal cavity, left orbit, nasopharynx, the choane and maxillary sinus left and extended to the tonsillar fossa without lysis of the skull base. We performed a biopsy of the lesion in the fossa nasal by nasal endoscopy. 


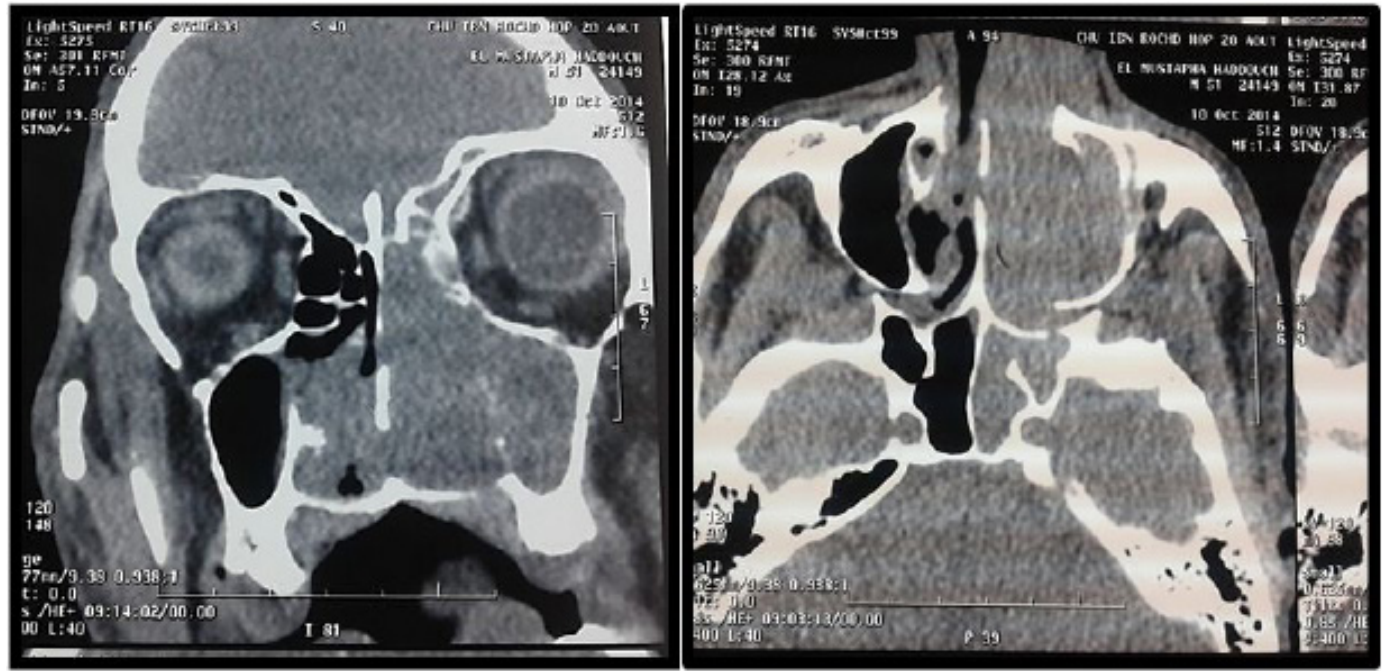

Figure 1: Frontal and transverse section showing mass occupying both nasal cavities, with destruction of the lamina papyracea of the left nostril and involvement of the left orbit, extending into maxillary sinus and prolapsed in the oropharynx
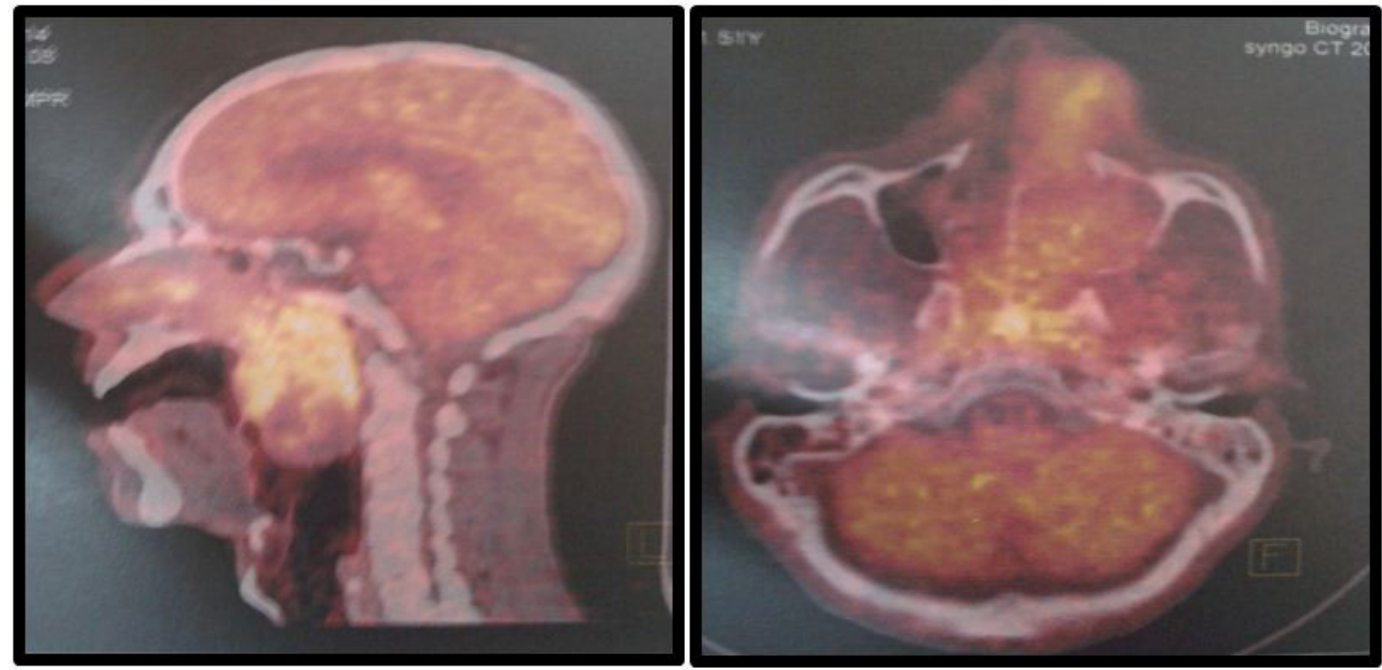

Figure 2: Sagittal and frontal section showing a hypermetabolic mass occupying the nasal cavity, left orbit, nasopharynx, the choane and maxillary sinus left and extended to the tonsillar fossa without lysis of the skull base.

The microscopic study indicated a proliferation of large cell layers, abundant eosinophilic cytoplasm and irregular hyper chromic nucleus. There were areas of necrosis with figures of typical and atypical mitoses. Immuno-histochemical analysis showed negativity with anti-cytokeratin antibodies, anti-desmin and anti-myogenin. The definitive diagnosis was poorly differentiated ostéosarcoma of the nasal fossa (Figure 3).

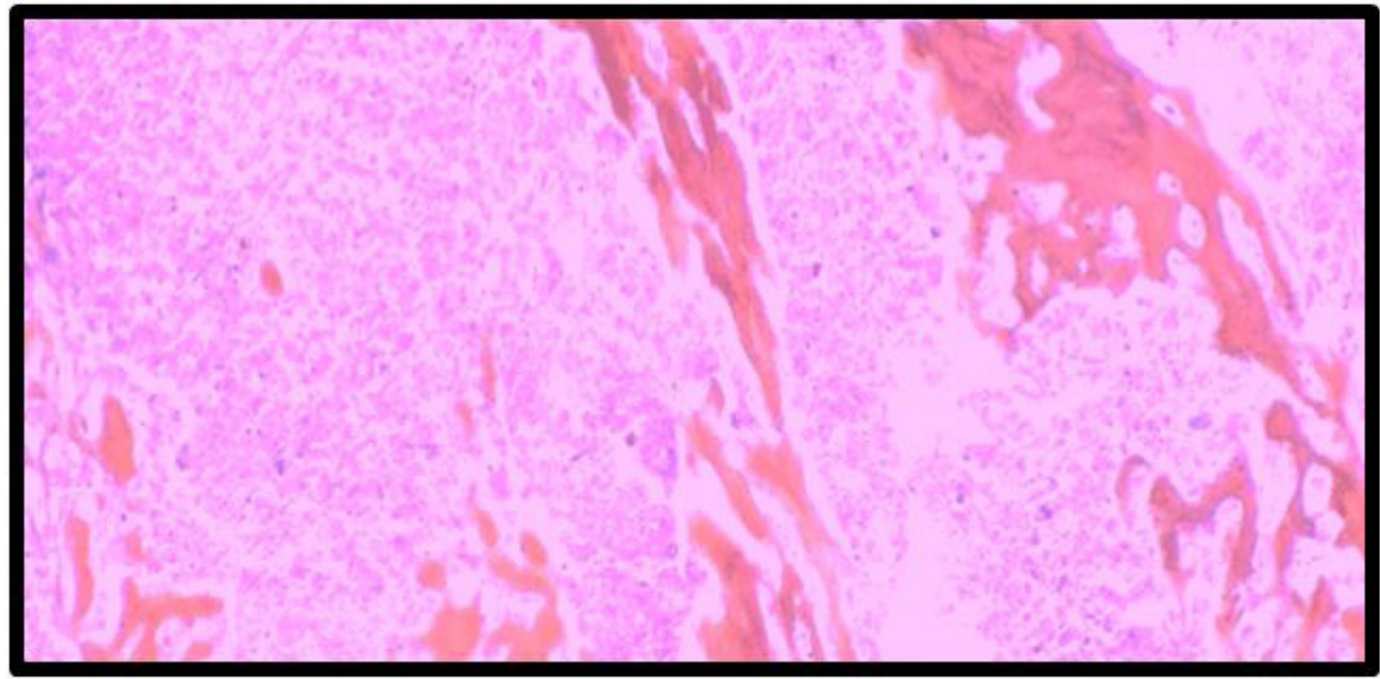

Figure 3: Proliferation of large cell layers with areas of necrosis with figures of typical and atypical mitoses. 
The multidisciplinary board meeting decision consisted neoadjuvant chemotherapy of (adriblastine, cisplatin and ifosfamide) given the extent of the tumor to nasopharynx and the impossibility of R0 surgery.

After 4 cycles of chemotherapy, the response was $80 \%$ of the initial tumor according to RECIST criteria (Figure $4-5$ ). The evolution was marked by an improvement of visual acuity. But our patient refused surgery.
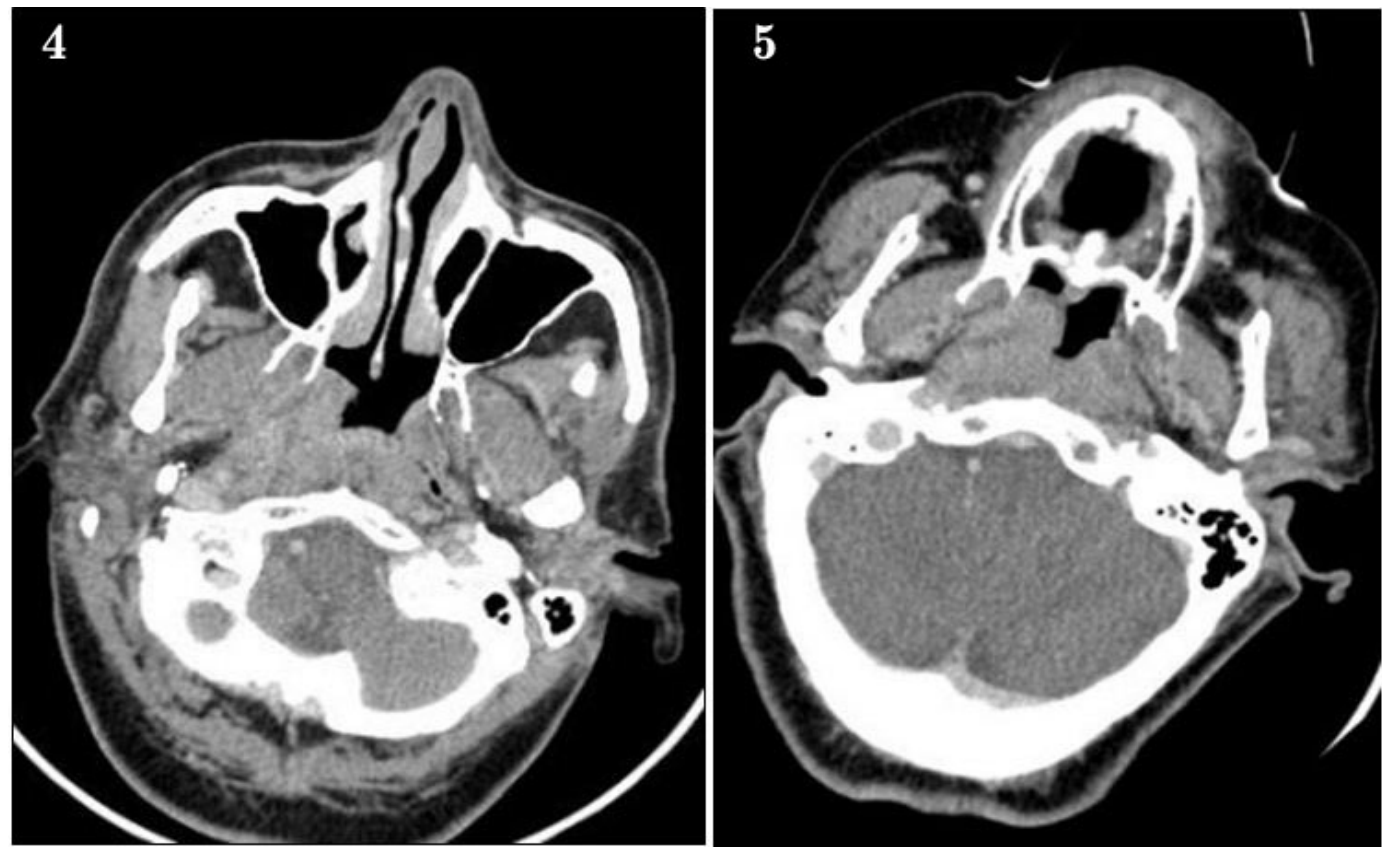

Figure 4-5: After 4 cycles of chemotherapy, Decrease of tumor volume the response was $80 \%$ of the initial tumor according to RECIST criteria

Given the characteristics of the tumor, the patient underwent radiotherapy treatment, being administered a total dose of 70 Gy with fractionation of 2 Gy/day, 5 days/week, with 5 photon fields of $6 \mathrm{MV}$. The first volume 70 Gy included the macroscopic tumor pre chemotherapy with margin of $5 \mathrm{~mm}$ which reduced to 1 or $2 \mathrm{~mm}$ near the critical organ like the brain or marrow stem. And the second volume $50 \mathrm{~Gy}$ include the 70 with margin of $3 \mathrm{~mm}$, ethmoid sinus on both side, lower frontal, sinus sphenoid, all the nasopharynx, the maxillary sinus homolateral, ipsilateral tonsillar, lodge ipsilateral infratemporal fossa and ipsilateral masticatory space saw the achievement of the medial pterygoid.

Due to the complicated anatomic relationship between the tumor and normal structures in the head and neck, and the importance of organ preservation in maintaining the patient's quality of life, the patient was treated by $3 \mathrm{D}$ conformal radiation therapy (3D-CRT), which allows highly conformal dose distributions to target volumes of almost any shape, appropriate selection and accurate delineation of the target volumes and the avoidable organs becomes of critical importance like the brainstem, the eye and optic nerve in our case.

Monthly checks were performed and the patient once again referred a drop of visual acuity in his left eye after radiotherapy. After 18 months of follow-up, there are no signs of recurrence or metastasis.

\section{Discussion}

Osteosarcomas of the head and neck represent a small percentage of all osteosarcomas with studies reporting incidence between $0.5-8.1$ percent $[2,3]$. These tumors usually present in the second to third decades of life, later than the presentation of osteosarcoma arising in the long-bone and usually occur as secondary tumors after radiation therapy, thorium oxide exposure, chemotherapy, inherited predispositions to development of osteosarcoma, or arise from a preexisting benign bone disease such as Paget's disease, bone infarcts, osteomyelitis, trauma [4-6]. No gender predominance has been described [7-9]. The most frequent site of location for craniofacial osteosarcomas is the mandible and maxilla [7,10-12].

The treatment protocols of osteosarcoma is based on the large trials or meta-analysis which are conducted for the limbs and the trunk, notably many of these studies have omitted head and neck region due to complexities of treatment in these site, moreover; head and neck osteosarcomas have not showed a similar pattern of demographic presentations of the other sites of the body [13]. The mean age of diagnosis of head and neck osteosarcoma is 30 years of age while children and adolescents are most often affected for other sites. The survival outcomes of osteosarcoma among various sites and age groups vary drastically and because of these differences, the treatment protocols of other sites are not applicable in head and neck sites [14].

The mainstay of treatment of head and neck osteosarcoma is surgery [15]. Adjuvant postoperative RT is indicated for those with close or positive margins and in high grade [16]. 
The role of chemotherapy, in particular neoadjuvant chemotherapy, is controversal. Most studies showed that chemotherapy has important place in the treatment of sarcomas of the long bones and has undoubtedly improved long-term, recurrence-free survival [15]. Whether this can be extrapolated to osteosarcomas of head and neck is a subject of debate. Because of the intact blood supply preoperatively there is speculation that neoadjuvant chemotherapy reduces the cellular viability of the peripheral tumor, which reduces the chance of invaded margins, and decreases the risk of local relapse and the number of eventual lung metastasis $[17,18]$.

Recently, chemotherapy directed by targeted nanoparticulate drug delivery system represents a promising approach for osteosarcoma treatment, using RGD peptide-installed doxorubicin-loaded biodegradable polymeric micelle,or by 2-Methoxyestradio, it's a physiological metabolite of $17 \beta$-estradiol, cause cell cycle arrest and apoptosis in osteosarcoma cells $[19,20]$.

The prognosis seems to depend on location (better in the upper aerodigestive tract), size (better for those less than $5 \mathrm{~cm}$ ) extension (better for those without bone extension) [3,21]. There are various cases in the literature with a good outcome, which are free from disease after monitoring for many years [22].

It was reported cases of Ewing's sarcoma and synovial sarcoma in almost all locations of the head and neck area, including the nasopharynx, tonsils, oropharynx, thyroid gland and parapharyngeal space. Nasal and maxillary involvement is particularly rare. In the literature, there is an apparent relationship with a history of head and neck radiation $[1,23,24]$.

To our knowledge we report here the first case of osteosarcoma of nasal fossa of 54-years- old occurred in a patient older than the average found in the literature, who hasn't any predisposition factors. After following the treatment, just chemotherapy and radiotherapy, the patient is currently free from tumor, 18 months later.

\section{Conclusion}

Despite the low incidence of this neoplasm in the head and neck region (less than 5\% of sarcomas in this area) and the rarity of this location, we should report any cases found, in order to determine the clinical, epidemiological and prognostic characteristics of the tumor more accurately, as well as its appropriate treatment.

\section{References}

1. Mahmoud O, Beck R, Kalyoussef E, Chan Park R, Baredes S, et al. (2017) Adjuvant therapies utilization pattern and survival outcomes in high-grade head and neck soft tissue sarcoma; a population based study. Oral Oncology 66: 28-37.

2. Bozdogan Arpaci R, Yuyucu Karabulut Y, Kara T, Serinsoz Linke E, Gönlüșen G, et al. (2015) Osteosarcoma of the Sinonasal Cavity in a Child. Oral Surg Oral Medi Oral Pathol Oral Radiol 119: e127.

3. Gadwal SR, Gannon FH, Fanburg-Smith JC, Becoskie EM, Thompson LD (2001) Primary osteosarcoma of the head and neck in pediatric patients: a clinicopathologic study of 22 cases with a review of the literature. Cancer 91: 598- 605.

4. Kirby EJ, et al. (2011) Primary osteosarcoma of the skull. J Craniofac Surg 22: 2399-405.

5. Kanazawa R, Yoshida D, Takahashi H, Matsumoto K, Teramoto A (2003) Osteosarcoma arising from the skull-case report. Neurol Med Chir 43: 88-91.

6. Kim YJ, Kim Ju Y, Chu CY, Lee WJ, Jeon SY et al. (2012) Primary osteosarcoma arising from the middle turbinate in a pediatric patient. Clin Exp Otorhinolaryngol 5: $237-9$.

7. Vlychou M, Ostlere SJ, Kerr R, Athanasou NA (2007) Low-grade osteosarcoma of the ethmoid sinus. Skeletal Radiol 36: 459-62.

8. Kohanawa R, Tabuchi K, Okubo H, Nagata M, Hara A (2005) Primary osteogenic sarcoma of the ethmoid sinus: a case report. Auris Nasus Larynx 32: 411-3.

9. Chaudhary M, Chaudhary DS (2012) Osteosarcomas of the jaws. J Oral Maxillofac Pathol 2012: 16: 233-8.

10. Park YK, Ryu K, Park RH, Kim WD (2003) Low-grade osteosarcoma of the maxillary sinus. Skeletal Radiol 32: 161-4.

11. Van den Berg H, Schreuderal WH, de Lange J (2013) Osteosarcoma: A Comparison of Jaw versus Nonjaw Localizations and Review of the Literature. Sarcoma 2013: 316123 .

12. Huvos AG, Sundaresan N, Bretsky SS, Butler A (1985) Osteogenic sarcoma of skull: a Clinicopathologic study of 19 patients. Cancer 56: $1214-21$.

13. DC Allison, SC Carney, ER Ahlmann, A Hendifar, S Chawla, et al. (2012) A meta-analysis of osteosarcoma outcomes in the modern medical era. Sarcoma 2012: 704872 .

14. PK Ha, DW Eisele, FJ Frassica, Zahurak ML, McCarthy EF (1999) Osteosarcoma of the head and neck: a review of the Johns Hopkins experience. Laryngoscope 109: 964-9.

15. BA Guadagnolo, GK Zagars, AK Raymond, Benjamin RS, Sturgis EM (2009) Osteosarcoma of the jaw/craniofacial region: outcomes after multimodality treatmen. Cancer 115: 3262-70.

16. RJ Mark, JA Sercarz, L Tran, Dodd LG, Selch M et al. (1991) Osteogenic sarcoma of the head and neck. The UCLA experience. Arch Otolaryngol Head Neck Surg 117: 761-6.

17. S Sefiani, Amarti A, Boulaadas M, Maher M, Saidi A (2005) Synovial sarcoma of the head and neck: two cases report. Rev Laryngol Otol Rhinol (Bord) 126: 53-6.

18. G Rosen, B Caparros, AG Huvos, C Kosloff, A Nirenberg, et al. (1982) Preoperative chemotherapy for osteogenic sarcoma: selection of postoperative adjuvant: chemotherapy based on the response of the primary tumour to preoperative chemotherapy. Cancer 49: 1221-30.

19. Fang Z, Sun Y, Xiao H, Li P, Liu M, et al. (2017) Targeted osteosarcoma chemotherapy using RGD peptide-installed doxorubicin-loaded biodegradable polymeric micelle. Biomed Pharmacother 85: 160-8. 
20. Gorska M, Kuban-Jankowska A, Zmijewski M, Marino Gammazza A, Cappello F, et al. (2015) 2-methoxyestradiol (DNA strand breaks induced by nuclear hijacking of neuronal NOS as an anti-cancer effect of 2-methoxyestradiol. Oncotarget 6: 15449-63.

21. M Salvati, P Ciapetta, A Raco (1993) Osteosarcomas of the skull: clinical remarks on 19 cases. Cancer 71: 2210-6.

22. Al-Daraji, Wael, Lasota Jerzy, Foss Robert, Miettinen Markku (2009) Synovial sarcoma involving the head: analysis of 36 cases with predilection to the parotid and temporal regions. Am J Surg Pathol 33: 1494-1503.

23. Gonzalez EM, Raghavan P, Cho B, Muttikkal EJT, Rehm KP (2016) Primary osteogenic osteosarcoma of the ethmoid sinus in an adolescent: case report. J Radiol Case Rep 10: 1-9.

24. C Galy-Bernadoy, R Garrel (2016) Head and neck soft-tissue sarcoma in adults. Euro Annal Otorhinolaryngol Head Neck dise 133: 37-42.

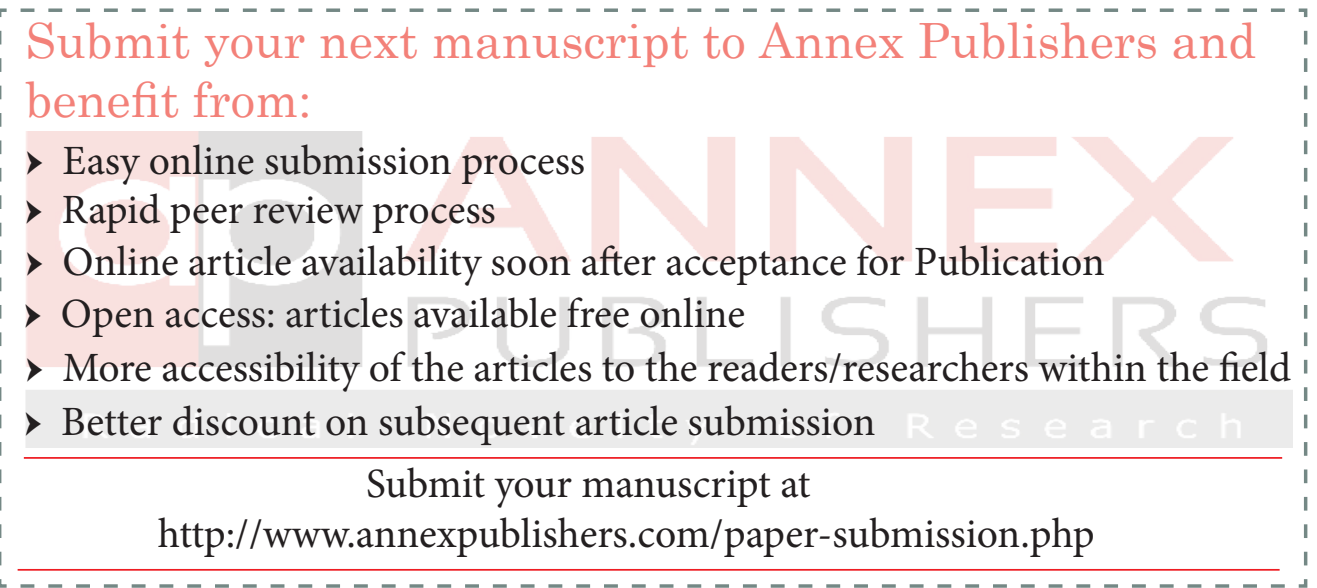

